\author{
Ramathasan Thevamaran \\ Division of Engineering and Applied Sciences, \\ California Institute of Technology, \\ Pasadena, CA 91125 \\ Department of Mechanical and \\ Process Engineering, \\ Swiss Federal Institute of Technology \\ (ETH Zurich), \\ Zurich 8092, Switzerland \\ e-mail: rthevama@caltech.edu \\ Fernando Fraternali \\ Department of Civil Engineering, \\ University of Salerno, \\ Fisciano, SA 84084, Italy \\ e-mail: f.fraternali@unisa.it \\ Chiara Daraio ${ }^{1}$ \\ Division of Engineering and Applied Sciences, \\ California Institute of Technology, \\ Pasadena, CA 91125: \\ Department of Mechanical and \\ Process Engineering, \\ Swiss Federal Institute of Technology \\ (ETH Zurich) \\ Zurich 8092, Switzerland \\ e-mail: daraio@ethz.ch
}

\section{Multiscale Mass-Spring Model for High-Rate Compression of Vertically Aligned Carbon Nanotube Foams}

We present a one-dimensional, multiscale mass-spring model to describe the response of vertically aligned carbon nanotube (VACNT) foams subjected to uniaxial, high-rate compressive deformations. The model uses mesoscopic dissipative spring elements composed of a lower level chain of asymmetric, bilateral, bistable elastic springs to describe the experimentally observed deformation-dependent stress-strain responses. The model shows an excellent agreement with the experimental response of VACNT foams undergoing finite deformations and enables in situ identification of the constitutive parameters at the smaller lengthscales. We apply the model to two cases of VACNT foams impacted at $1.75 \mathrm{~ms}^{-1}$ and $4.44 \mathrm{~ms}^{-1}$ and describe their dynamic response.

[DOI: $10.1115 / 1.4028785]$

Keywords: carbon nanotube foams, mechanical properties, high-rate compression, multiscale modeling, strain localization, hysteresis

\section{Introduction}

Macroscale carbon nanotube (CNT) foams have been synthesized from vertically aligned bundles of CNTs [1] or randomly oriented CNT fibers (sponges) [2]. Their exceptional mechanical properties and energy absorption characteristics make these standalone CNT-foams excellent candidates for various applications [3] including energy absorbing/protective packaging materials for electronics and mechanical systems [1,2], structural reinforcements in composites [4] and woven fibers for bulletproof tough textiles [5]. Bulk VACNT foams present a hierarchical fibrous microstructure with constituent features in various lengthscales (Ref. [1], Fig. 3 in Ref. [6]): The individual multiwalled carbon nanotubes (MWCNTs) have a concentric tubular configuration with several walls in the nanoscale; the MWCNTs entangle with each other to form a forest-like system in the microscale; and the bundles of MWCNTs are aligned vertically in the mesoscale. When subjected to compressive loadings, they exhibit distinct deformation mechanisms at different lengthscales: A foam-like compression in the macroscale; collective sequential buckling of the aligned CNT bundles in the mesoscale; and bending and buckling of individual tubes in the microscale [1,7]. The bulk compression response of VACNT foams is identified with three distinct loading regimes: An initial linear elastic regime, a plateau regime governed by progressive buckles and a final densification regime [1].

VACNT foams exhibit different mechanical responses when subjected to different loading regimes. Macroscale samples exhibit a viscoelastic response when subjected to long duration stress relaxation experiments in compression [8] or when tested for creep with nano-indentation [9]. The same material exhibits rate-independent deformation mechanisms in quasi-static compression experiments [10]. However, few studies suggested dependence of VACNT foam's unloading modulus and recovery on

\footnotetext{
${ }^{1}$ Corresponding author.

Contributed by the Applied Mechanics Division of ASME for publication in the Journal OF APPLIED MeChanICS. Manuscript received August 11, 2014; final manuscript received October 8, 2014; accepted manuscript posted October 13, 2014; published online October 27, 2014. Editor: Yonggang Huang.
}

strain rate $[11,12]$. In the linear dynamic regime, VACNT foams subjected to torsional mode dynamic mechanical analysis exhibited a frequency invariant dissipative response [13]. The VACNTs storage and loss moduli were shown to be independent of frequency in uniaxial linear vibration experiments [14]. VACNT foams impacted by a striker exhibit complex rate-effects: The loading response is rate-independent, whereas the unloading modulus increases with strain rate [7]. When VACNT foams are impacted at velocities higher than a critical velocity $\left(\sim 6.5 \mathrm{~ms}^{-1}\right)$, they support shock formation [7].

Several models have been proposed to describe the rateindependent mechanical response of VACNT foams in the quasistatic regime. Analytical micromechanical models supported by finite element models have been used to describe the response of forests of VACNTs subjected to nano-indentation with a spherical indenter [15]. It has been shown that the indentation force during nano-indentation scales linearly with tube areal density, tube moment of inertia, tube modulus, and indenter radius, whereas the force scales inversely with the square of tube length [15]. Buckle's formation and progression in VACNT micropillars under quasi-static compression has been modeled using a finite element formulation of an isotropic viscoplastic solid combined with piecewise hardening-softening-hardening function [16]. It revealed that the buckle wavelength decreases with increasing magnitude of "negative hardening slope" and the buckle wave amplitude increases with the increasing width of the flow strength function well [16]. It was also found that the buckles always initiated near the substrate due to the displacement constraint and sequentially progressed even in the absence of a property gradient along the height of the sample [16]. Recently, a Timoshenko beam model for an inelastic column in buckling has been used to predict the critical buckling stress of VACNT micropillars with transverse isotropy [17].

Coarse-grained molecular dynamic simulations of VACNT foams [18] have found that the frequency-independent viscoelasticity in shearing [13] arises from rapid unstable attachment/ detachment among individual CNTs induced by the van der Waals forces and contributes to constantly changing microstructure of the CNT network. This rate-independent dissipation was also 
described using triboelastic constitutive models and it has been shown that the increased adhesive energy significantly increased the overall stiffness of the network compared to the tension-, bending-, and torsion-stiffnesses. This suggests that the van der Waals interaction not only contributes to energy dissipation but also influences the elasticity of the network [18]. A phenomenological multiscale mass-spring model with bistable elements has been used to describe the rate-independent quasi-static compressive response of macroscale VACNT foams [19]. This model also enabled in situ material parameter identification in multilayered CNT arrays, and allows modeling experimentally observed local deformations accurately [20]. It has been extended later to describe a few experimentally observed phenomena, such as preconditioning [20], loading history, and loading direction dependency [10] and permanent damage [21]. However, numerical models of high-rate, uniaxial, finite deformation of VACNT foams have not yet been developed.

In this article, we propose a phenomenological mass-spring model that uses rate-independent, dissipative spring elements in association with phenomenological damping devices [22] to describe dynamic response of bulk VACNT foams. We use this model to describe the global dynamic response observed in experiments and then to identify the deformation-dependent microscale mechanical parameters in situ. In the following sections, we provide a detailed description of the experimental methods and observations (Sec. 2), a detailed description of the generalized mechanical model (Sec. 3) and the application of this model to describe the dynamic response of VACNT foams with in situ parameter identification (Sec. 4).

\section{Brief Overview of Experimental Methods and Observations}

Dynamic experiments were performed on an impact testing setup using a flat plunge striker as the loading apparatus. The complete description of the experimental setup and the data analysis methodologies can be found in Ref. [6]. The VACNT foam samples were attached to a striker and launched at controlled velocities on a frictionless guide to directly impact a force sensor. A rigidly mounted force sensor recorded the transmitted forcetime history during impact, while a geometric moiré transducer measured the dynamic deformation. These measurements were then used to obtain the dynamic stress-strain diagram, from which dynamic constitutive parameters were calculated. A high-speed microscopic camera was used for in situ visualization and characterization of the microscale complex deformations [6].

When a VACNT foam sample is impacted, the stress rises nonlinearly with strain up to the peak stress, corresponding to the maximum strain. In unloading, the stress decreases rapidly within the first $10 \%$ of the unloading strain, and gradually reaches zero. The stress-strain hysteresis loop formed by the loading-unloading cycle represents the energy dissipated during the dynamic compression. In situ visualization using a high-speed microscopic camera reveled formation and progression of sequential buckle instabilities in the sample during the loading phase (see supplementary video in Ref. [7]). The synthesis of VACNT foams, achieved using a chemical vapor deposition (CVD) process, induces an intrinsic density gradient along the height of the samples $[1,23]$. Synchrotron X-ray scattering and mass attenuation measurements showed that this intrinsic density gradient is nonlinear in a sample synthesized using a floating-catalyst CVD process and presents a lower density region near the growth substrate and denser region near the free surface of the sample [7]. Because of this characteristic anisotropic microstructure, buckles always nucleate at the bottom of the soft region and progress sequentially toward the stiffer region [7]. The formation and progression of instabilities is reflected also in the dynamic stress-strain diagram and the stress-time histories, and it is evident from local stress drops followed by local stiffening [6,7]. Upon unloading, the buckles sequentially recover [6,7]. When the samples are impacted repeatedly, with increasing impact velocity, they exhibit a preconditioning effect, which is evident from the different loading paths measured during each consecutive cycle [7]. The loading response is independent of the impact velocity, while the unloading is rate-dependent [7]. The unloading modulus was observed to increase with the increasing strain rate (Fig. 1(b) in Ref. [7]). The dynamic loading stress-strain response of VACNT foams is similar to their response in quasi-static compression until reaching a critical impact velocity $\left(\sim 6.5 \mathrm{~ms}^{-1}\right)$ [7]. When the samples are impacted with striker velocities higher than the critical velocity, the formation of progressive buckles transitions into the formation and propagation of a shock [7].

In this article, we model only the response of samples impacted at subcritical velocities. We consider two samples, with similar bulk densities, that were impacted by a striker with mass $7 \mathrm{~g}$ at two different impact velocities: $1.75 \mathrm{~ms}^{-1}$ (VACNT foam-1) and $4.44 \mathrm{~ms}^{-1}$ (VACNT foam-2). The physical properties of the two samples are summarized in Table 1. Both samples exhibited a full recovery of the deformation upon unloading. We use experimental force-time histories to prescribe load-histories in the model, and we calculate the dynamic responses during the time the sample is in contact with the force sensor. In Sec. 3, we present the numerical model in details.

\section{Mechanical Model}

We use a one-dimensional, multiscale, phenomenological model to numerically describe the dynamic response observed experimentally (and summarized in Sec. 2). The model describes the response of VACNT foams at the mesoscopic scale, through the discretization of the foams into a collection of lumped masses connected by dissipative springs [19]. Each mesoscopic spring represents the continuum limit of a chain of infinitely many microscopic bistable elastic springs. The bistable springs are characterized by two stable phases (prebuckling loading and postbuckling densification) and an intermediate unstable phase (buckling phase). The dynamic snapping of the microscopic springs and the subsequent snapping back induce hysteretic energy dissipation via "transformational plasticity" [19,24]. Our model comprises two different timescales: An external timescale, which controls the evolution of the applied loading and the response at the mesoscale; and an internal timescale, which governs the dynamic relaxation of the system at the microscale, for a fixed external time. The constitutive behavior is viscous at the microscale, and rate-independent at the mesoscale $[19,24]$. Eventually, the overall response of a CNT structure can be described through a single dissipative element (macroscopic mass-spring model $[10,25])$. This multiscale model has been previously applied to describe the quasi-static response of CNT structures $[10,19,20,21,25]$. Here, the same model is applied to describe the mechanical response of VACNT foams under high-rate loading in association with the phenomenological damping devices [22]. The constitutive parameters are assigned depending on the applied strain rate. Alternatively, rate-dependent models that accounts for the evolution laws of material parameters as a function of strain rate can be formulated, and we leave such an extension of the model for the future work.

We briefly summarize the analytic formulation of the model at the mesoscale, which is detailed in Ref. [19]. Let us introduce a chain of $N+1$ lumped masses $m^{0}, \ldots, m^{N}$, connected by $N$

Table 1 Physical properties of the VACNT foam samples

\begin{tabular}{lcc}
\hline \hline & VACNT foam-1 & VACNT foam-2 \\
\hline Mass $(\mathrm{mg})$ & 5.56 & 5.05 \\
Diameter $(\mathrm{mm})$ & 5 & 5 \\
Height $(\mathrm{mm})$ & 1.190 & 1.106 \\
Bulk density $\left(\mathrm{g} \mathrm{cm}^{-3}\right)$ & 0.238 & 0.232 \\
\hline \hline
\end{tabular}


nonlinear spring elements $(N \geq 1)$. The mass $m^{0}$ is clamped at the bottom (fixed-boundary), at position $x^{0}=0$, and the mass $m^{N}$ is free at the top (free-boundary), at position $x^{N}=l$. Spring 1 is at the bottom and connects masses $m^{0}$ and $m^{1}$ while spring $N$ is at the top and connects $m^{N}$ and $m^{N+1}$. The scalar quantity, $\varepsilon^{i}$ characterizes the total strain at the $i$ th spring

$$
\varepsilon^{i}=\frac{u^{i-1}-u^{i}}{h^{i}}
$$

where $u^{i}$ is the axial displacement of the mass $m^{i}$ relative to its initial position and $h^{i}=x^{i}-x^{i-1}$. The constitutive equations for each mesoscopic spring are

$$
\sigma^{i}=\left\{\begin{array}{l}
\sigma^{(a, i)}=k_{0}^{i} \varepsilon^{i} /\left(1-\varepsilon^{i}\right), \quad \text { for } \quad\left(\varepsilon^{i}<\hat{\varepsilon}_{a}^{i}\right) \quad \text { or } \quad\left(\hat{\varepsilon}_{a}^{i}<\varepsilon^{i}<\varepsilon_{a}^{i}\right) \quad \text { and } \quad\left(\operatorname{flag}^{(k-1)} \neq c\right) \\
\sigma^{(d, i)}=\sigma_{a}^{i}+k_{h+}^{i}\left(\varepsilon^{i}-\varepsilon_{a}^{i}\right), \quad \text { for } \quad\left(\varepsilon_{a}^{i} \leq \varepsilon^{i} \leq \hat{\varepsilon}_{c}^{i}\right) \quad \text { and } \quad\left(\operatorname{flag}^{(k-1)}=a\right) \\
\sigma^{(e, i)}=\sigma_{a}^{i}+\Delta \sigma^{i}+k_{h-}^{i}\left(\varepsilon^{i}-\bar{\varepsilon}_{c}^{i}\right), \quad \text { for } \quad\left(\hat{\varepsilon}_{a}^{i} \leq \varepsilon^{i} \leq \bar{\varepsilon}_{c}^{i}\right) \quad \text { and } \quad\left(\operatorname{flag}^{(k-1)}=c\right) \\
\sigma^{(c, i)}=k_{c}^{i}\left(\varepsilon^{i}-\varepsilon_{*}^{i}\right) /\left(1-\left(\varepsilon^{i}-\varepsilon_{*}^{i}\right)\right), \quad \text { for }\left(\varepsilon^{i}>\hat{\varepsilon}_{c}^{i}\right) \quad \text { or } \quad\left(\bar{\varepsilon}_{c}^{i}<\varepsilon^{i}<\hat{\varepsilon}_{c}^{i}\right) \quad \text { and } \quad\left(\mathrm{flag}^{(k-1)} \neq a\right)
\end{array}\right.
$$

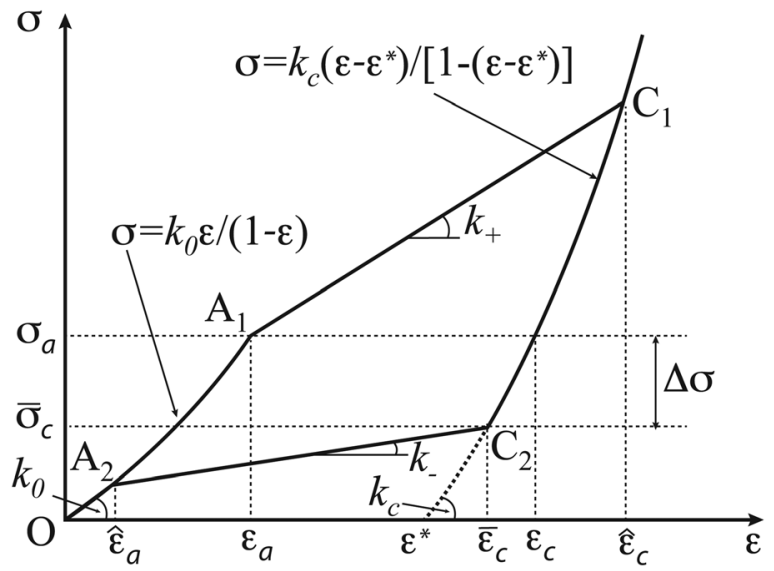

Fig. 1 Schematic diagram showing the response of a generic mesoscopic dissipative spring element and the relevant constitutive parameters
Here, $\sigma^{i}$ is the stress at each time step $t=t_{k}(k=1, \ldots, M)$ and

$$
\text { flag }^{(k)}=\left\{\begin{array}{l}
a, \quad \text { if } \quad \sigma^{i}=\sigma^{(a, i)} \\
c, \quad \text { if } \quad \sigma^{i}=\sigma^{(c, i)} \\
\text { flag }^{(k-1)}, \quad \text { otherwise }
\end{array}\right.
$$

The constitutive parameters $k_{0}^{i}, k_{c}^{i}, \Delta \sigma^{i}, \varepsilon_{a}^{i}, \varepsilon_{c}^{i}, k_{h+}^{i}$, and $k_{h-}^{i}$ in Eq. (2) are seven independent quantities, while $\hat{\varepsilon}_{a}^{i}$ and $\hat{\varepsilon}_{c}^{i}$ are computed by solving the following Eqs. (4) and (5) for $\varepsilon^{i}$, respectively,

$$
\begin{aligned}
& \sigma^{(a, i)}=\sigma^{(e, i)} \\
& \sigma^{(c, i)}=\sigma^{(d, i)}
\end{aligned}
$$

The stiffness parameters $k_{0}^{i}$ and $k_{c}^{i}$ represent the initial slopes $d \sigma^{i} / d \varepsilon^{i}$ at $\sigma^{i}=0$, of the bilateral branches $\mathrm{OA}_{1}$ and $\mathrm{C}_{1} \mathrm{C}_{2}$ (Fig. 1). These two branches represent the initial elastic regime and the final densification regime of each spring, respectively. The $k_{h+}^{i}$ is the slope of the unilateral branch $\mathrm{A}_{1} \mathrm{C}_{1}$, describing the snap (a)

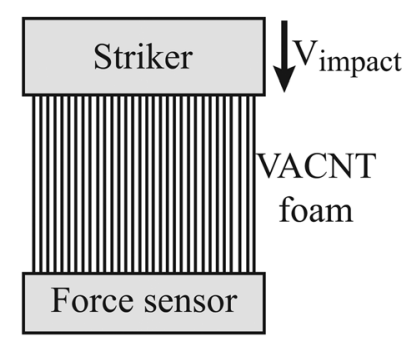

(c)

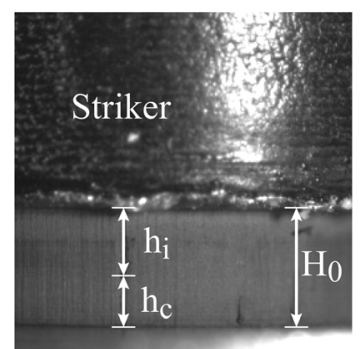

Pristine state (b)
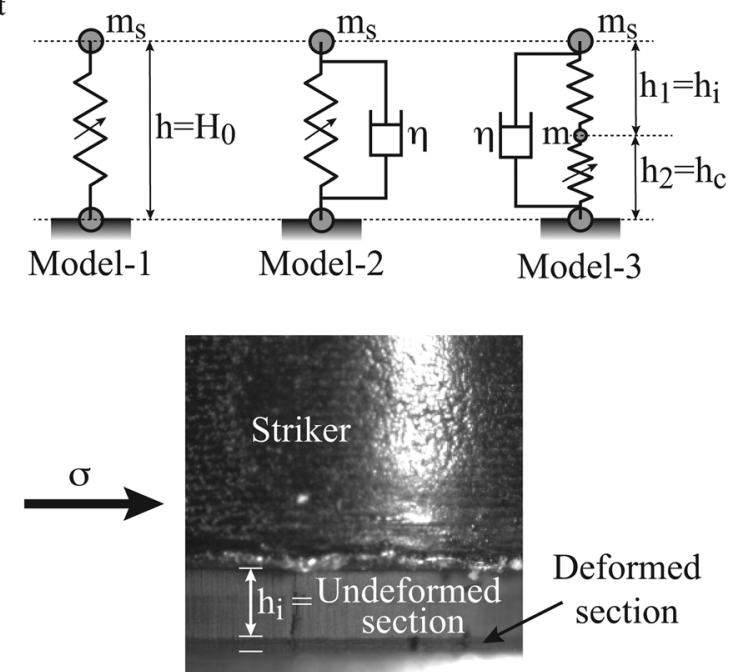

Deformed state

Fig. 2 Description of the model for the sample impacted at $1.75 \mathrm{~ms}^{-1}$. (a) Schematic of the experiment showing the sample being compressed by the striker against the rigidly mounted force sensor. (b) Three different models considered for the sample. (c) Optical images showing the pristine and deformed states of the sample [7]. Markers are used to highlight the deformed and undeformed sections of the sample. 
buckling and the consequent hardening during the loading phase. The $k_{h-}^{i}$ is the slope of the unilateral branch $\mathrm{C}_{2} \mathrm{~A}_{2}$, describing the snap-back recovery of the buckles during unloading phase. When $k_{h+}^{i}$ and $k_{h-}^{i}$ are zero, the unilateral branches describe a perfectly plastic behavior. The $\Delta \sigma^{i}$ equals to the $\bar{\sigma}_{c}^{i}-\sigma_{a}^{i}$, where the $\sigma_{a}^{i}$ and $\bar{\sigma}_{c}^{i}$ are the stresses corresponding to the points $\mathrm{A}_{1}$ and $\mathrm{C}_{2}$.

This model does not allow for accumulation of permanent strains that is often found in the compression experiments of VACNT foams, both in their quasi-static [1] and dynamic [7] responses. However, it can be modified to prevent snap-back recovery of springs and allow permanent damage [21]. Similarly, the model can be generalized to describe preconditioning effects found in cyclic loading, by introducing initial strains, $\varepsilon_{0}^{i} \geq 0$ and elastic strains $\underline{\varepsilon}^{i}=\varepsilon^{i}-\varepsilon_{0}^{i}$ for each spring as described in Ref. [20]. In this article, we will not attempt to extend these features in dynamics.

\section{Experimental Fit and In Situ Parameter Identification}

We model the striker as a rigid particle with lumped mass equal to the mass of the striker $(7 \mathrm{~g})$ and the force sensor as a rigid fixed wall (Figs. 2(a) and 2(b)). We apply the experimental stress-time history to the particle that represents the striker (top particle), and determine the stress-time and the displacement-time histories at the base of the VACNT foam (force sensor side) using the numerical model described in Sec. 3. The whole sample is assumed to be in dynamic equilibrium throughout the experimental duration [6].

Figure 2(c) shows selected snap-shots obtained from the highspeed image sequence, corresponding to the pristine state of the VACNT foam-1 at the instance of impact $\left(V_{\text {striker }}=1.75 \mathrm{~ms}^{-1}\right)$ and the deformed state at maximum compression $\left(V_{\text {striker }}=0\right)$. A visualization of the dynamic deformation of the sample can be found in the supplementary video of Ref. [7]. As shown on Fig. 2(c), collective buckles nucleate at the bottom of the sample during impact and progressively compress the sample to the height of $h_{c}=0.490 \mathrm{~mm}$. The remaining section of the sample with height, $h_{i}=0.700 \mathrm{~mm}$ undergoes infinitesimal compressive strains. As a first approximation (model-1 in Fig. 2(b)), we represent the whole height $(1.190 \mathrm{~mm})$ of the sample as a single effective spring (macroscopic dissipative element) that connects the striker particle to the rigid wall (force sensor). In addition, we neglect the mass of the VACNT foam $(5.56 \mathrm{mg})$ in comparison to the large striker mass $(7 \mathrm{~g})$. The seven independent parameters

Table 2 Parameters of the models of VACNT foam-1, impacted at a velocity of $1.75 \mathrm{~ms}^{-1}$

\begin{tabular}{|c|c|c|c|c|c|c|c|c|c|c|}
\hline & & $k_{0}(\mathrm{MPa})$ & $\Delta \sigma / \sigma_{a}$ & $\eta(\mathrm{Pa} \mathrm{s})$ & $\epsilon_{a}$ & $\epsilon_{c}$ & $h(\mathrm{~mm})$ & $k_{h+} / k_{0}$ & $k_{h-} / k_{0}$ & $k_{c} / k_{0}$ \\
\hline Model-1 & & 5.50 & -0.20 & - & 0.14 & 0.31 & 1.190 & 2.60 & 0.450 & 15 \\
\hline Model-2 & & 5.50 & -0.20 & $1 \times 10^{4}$ & 0.14 & 0.31 & 1.190 & 2.60 & 0.425 & 15 \\
\hline \multirow[t]{2}{*}{ Model-3 } & S1 & 60 & - & $3 \times 10^{2}$ & - & - & 0.700 & - & - & - \\
\hline & S2 & 2 & -0.32 & & 0.35 & 0.71 & 0.490 & 3.40 & 0.525 & 30 \\
\hline
\end{tabular}

The definition of these parameters in shown in Fig. 3. In model-3, S1 is the linear spring and S2 is the nonlinear, bistable spring.
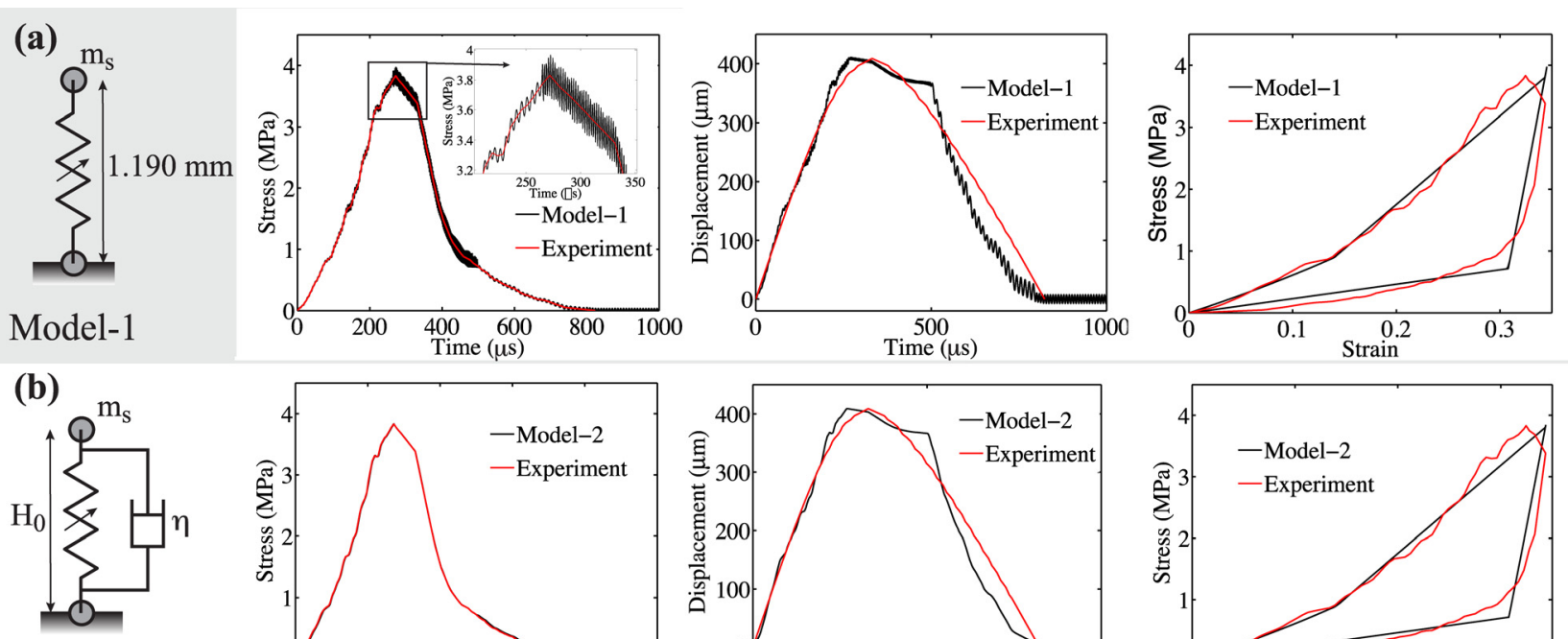

Model-2
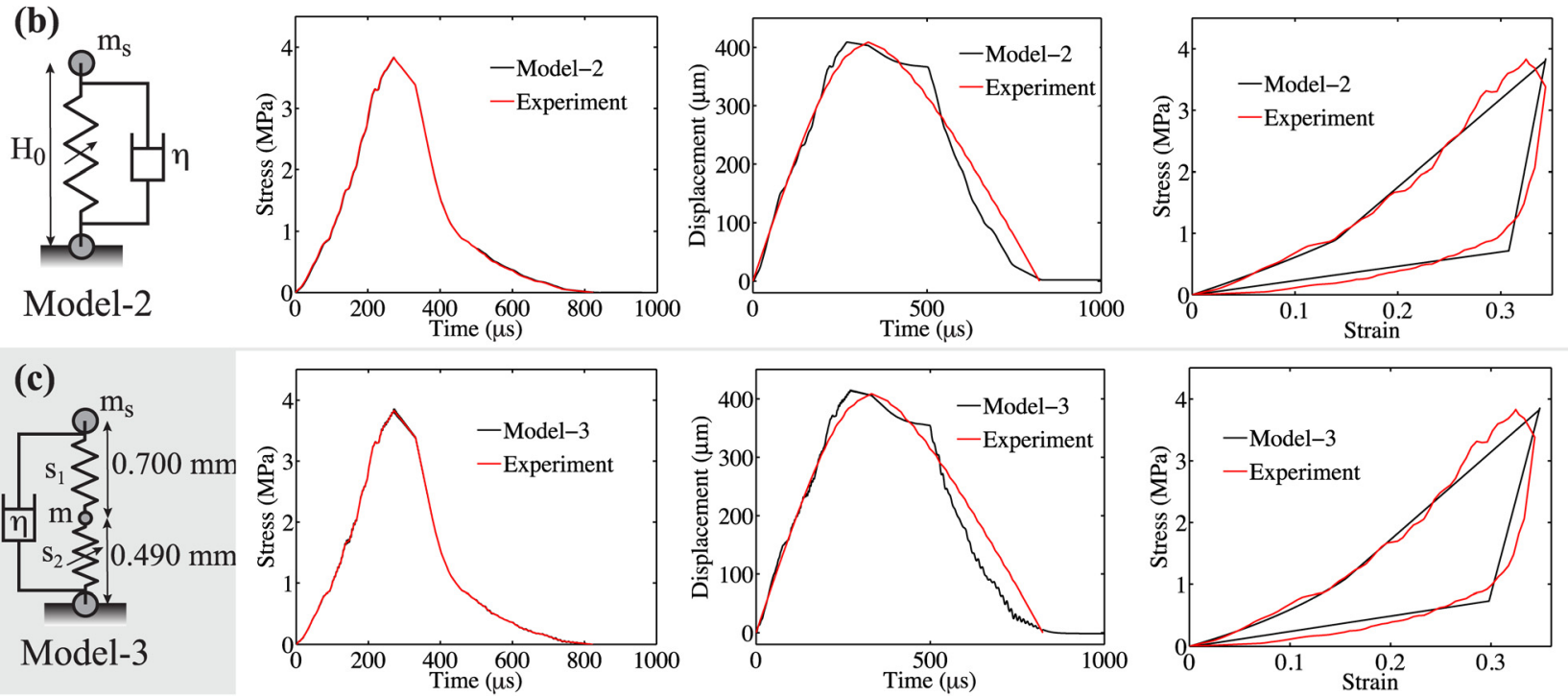

Fig. 3 Comparison of the numerical and experimental results of stress-time histories, displacement-time histories, and stress-strain response for (a) model-1, (b) model-2, and (c) model-3 of the VACNT foam-1 

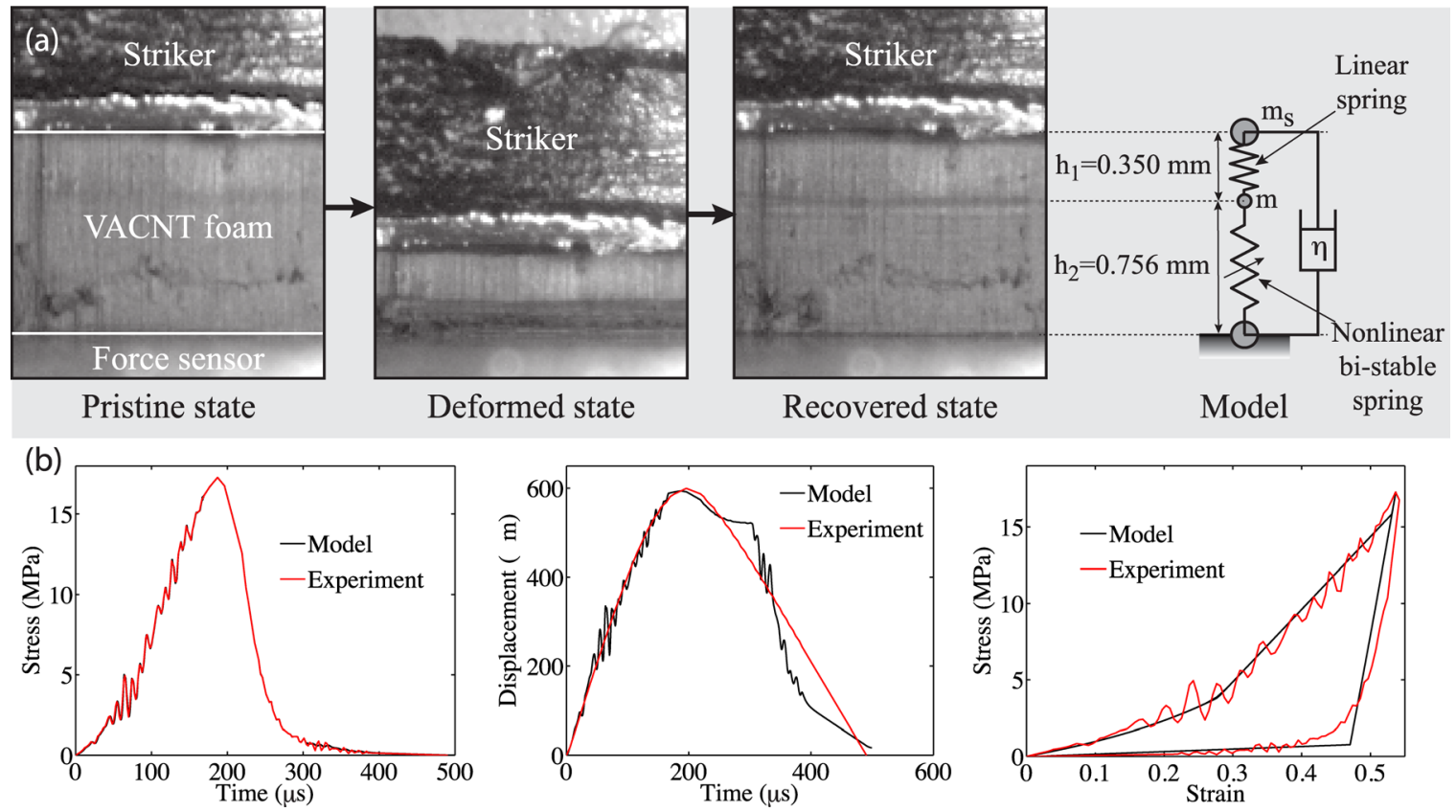

Fig. 4 (a) Optical images selected from the high-speed camera sequence showing the sample VACNT foam-2 before the impact (pristine state), at its maximum deformation (deformed state) and after load release (recovered state) [6]. The schematic diagram on the right shows the model employed and its relevant parameters. This sample was impacted at $4.44 \mathrm{~ms}^{-1}$. (b) Comparison of the numerical and experimental results for the stress-time history, displacement-time history and stress-strain response.

Table 3 Parameters of the model of VACNT foam-2, impacted at a velocity of $4.44 \mathrm{~ms}^{-1}$

\begin{tabular}{lccccccccc}
\hline \hline & $k_{0}(\mathrm{MPa})$ & $\Delta \sigma / \sigma_{a}$ & $\eta(\mathrm{Pa} \mathrm{s})$ & $\epsilon_{a}$ & $\epsilon_{c}$ & $h(\mathrm{~mm})$ & $k_{h+} / k_{0}$ & $k_{h-} / k_{0}$ \\
\hline S1 & 250 & - & $1 \times 10^{4}$ & - & - & 0.350 & - & $k_{c} / k_{0}$ \\
S2 & 5.75 & -0.80 & & 0.40 & 0.70 & 0.756 & 6.00 & 0.200 & 40 \\
\hline \hline
\end{tabular}

The definitions of these parameters are shown on the Figure 3. S1 is the linear spring and the S2 is the nonlinear bistable spring.

that define the nonlinear spring of model-1 are listed in Table 2. Figure 3(a) (top panel) shows the stress- and displacement-time histories and the stress-strain diagram obtained with model-1 (and superimposed to the experimental data). The overall results show a good agreement with experiments. The time histories of stress and displacement, however, exhibit significant oscillations that arise from numerical instabilities. These instabilities are particularly evident when the model transitions between adjacent branches of the dissipative spring element-for example, see the inset of stress-time history in Fig. 3(a). To ensure stability during the dynamic transitions between phases, we introduced an onsite damper with damping coefficient $0.01 \mathrm{MPa} s$ to the striker mass (model 2 in Fig. 2(b)). The damping ratio between the adopted damping coefficient and the critical damping coefficient associated with the unloading branch $\left(2 h \sqrt{\rho k_{c}}\right)$ is calculated to be 0.894. As shown in the middle panel of Fig. 3(b), the damper reduces the numerical instabilities significantly and facilitates smooth dynamic transitions.

We refine the model further to account for the elastic properties of the deformed section of the CNT foams (model-3). The refined model employs a dissipative spring element $\left(\mathrm{S}_{2}\right)$, with height $h_{2}=0.490 \mathrm{~mm}$, to describe the response of the heavily deformed (or "buckled") section of the sample, and models the section of the sample that undergoes infinitesimal strains through a linear spring $\left(\mathrm{S}_{1}\right)$ with height $h_{1}=0.700 \mathrm{~mm}$ (Fig. $2(b)$ ). We set the stiffness of this linear spring to be approximately equal to the unloading stiffness $\left(k_{c}\right)$ of the nonlinear spring, in order to localize the deformation in $\mathrm{S}_{2}$. We introduce another lumped mass equal to the mass of the VACNT foam sample $(5.56 \mathrm{mg})$ in between these two springs. The bottom panel of Fig. 3(c) shows that the numerically obtained global dynamic response of the sample is in good agreement with experiments. As shown in the parameters listed in Table 2, the nonlinear spring $\left(\mathrm{S}_{2}\right)$ of model-3 exhibit lower initial stiffness $\left(k_{0}\right)$ compared to that in model-2, since it identifies specifically the buckled region as an effective spring. Also, due to the snap-buckle and the consequent densification in spring $\mathrm{S}_{2}$, the unloading stiffness parameter $k_{c}$ shows a higher value compared to that of model-2. The damping ratio required for ensuring the numerical stability of model-3 is 0.0297 (significantly less than the same value in model-2). As it is presented on the Table 2, the two-spring model allows the identification of mechanical parameters at a lengthscale that is much smaller than the sample size. The bulk response of the sample is the effective response of these two elements, masses and the associated damping device.

We apply a similar two-spring model to the VACNT foam-2 that was impacted at $4.44 \mathrm{~ms}^{-1}$ (Fig. 4). Similar to the previous case, we use a dissipative element as an effective spring for the buckled section of the sample $\left(h_{2}=0.756 \mathrm{~mm}\right)$ and represent the infinitesimally strained section $\left(h_{1}=0.350 \mathrm{~mm}\right)$ with a unilateral linear spring. An onsite damper with damping coefficient of $1 \times 10^{4}$ is used to ensure stability during numerical simulation. The damping ratio required for such numerical stability is 0.525 .

Figure $4(b)$ shows the comparison of numerical and experimental results. The model captures the global dynamics, while identifying the constitutive parameters at a lower lengthscale compared to the sample height (Table 3 ). It should be noted that the sample impacted at high velocity deforms more and reaches higher maximum strain, as compared to VACNT foam-1. Since the height of the nonlinear spring $\left(S_{2}\right)$ describing the buckled region is 
significantly higher in VACNT foam-2 than in VACNT foam-1, the stiffness constant $k_{0}$ of VACNT foam-2 (5.75 MPa) is appreciably higher than $k_{0}$ of VACNT foam-1 $(2.00 \mathrm{MPa})$. This increase in stiffness is explained by the increase in the intrinsic density of CNTs along the height [7].

\section{Conclusions}

We introduced phenomenological models to describe the dynamic response of VACNT foams under high-rate compression. The models use a one-dimensional mass-spring system containing an effective dissipative spring element, which describes either the entire sample (single-spring model), or its buckled (heavily deformed) section (two-spring model). We have shown that the models allow us to characterize the bulk dynamic response of the VACNT foams and their dissipation properties. The adopted spring models employ the concept of rate-independent, transformational plasticity, as opposed to more conventional, ratedependent and/or plastic models. We showed that such models could be generalized to high-rate compression responses when they are used in association with damping devices, and the material parameters are modulated according to the applied strain rate. We introduced numerical viscosity through the phenomenological approach proposed in Ref. [22]. The two-spring model enables the identification of the VACNT foams' deformation-dependent mechanical parameters, at lengthscales smaller than the sample height, which cannot be obtained alone from the experimental measurements. Even though we use our model to describe the VACNT foam's dynamic responses, the model can be extended to other hierarchical materials, with fibrous morphology. Additional future research lines include the formulation of evolution laws of the material parameters as a function of the applied strain rate, and the numerical modeling of large-scale problems through the quasicontinuum method and local maximum-entropy schemes [26,27].

\section{Acknowledgment}

We acknowledge financial support from the Institute for Collaborative Biotechnologies (ICB) under the Contract No. W911NF-09-D-0001 with the Army Research Office (ARO). F.F. acknowledges financial support from the Italian Ministry of Foreign Affairs, Grant No. 00173/2014, Italy-USA Scientific and Technological Cooperation 2014-2015 ('Lavoro realizzato con il contributo del Ministero degli A ari Esteri, Direzione Generale per la Promozione del Sistema Paese').

\section{References}

[1] Cao, A., Dickrell, P. L., Sawyer, W. G., Ghasemi-Nejhad, M. N., and Ajayan, P. M., 2005, "Super-Compressible Foamlike Carbon Nanotube Films," Science, 310(5752), pp. 1307-1310.

[2] Gui, X., Wei, J., Wang, K., Cao, A., Zhu, H., Jia, Y., Shu, Q., and Wu, D., 2010, "Carbon Nanotube Sponges," Adv. Mater., 22(5), pp. 617-621.

[3] De Volder, M. F. L., Tawfick, S. H., Baughman, R. H., and Hart, A. J., 2013, "Carbon Nanotubes: Present and Future Commercial Applications," Science, 339(6119), pp. 535-539.

[4] Ci, L., Suhr, J., Pushparaj, V., Zhang, X., and Ajayan, P. M., 2008 "Continuou Carbon Nanotube Reinforced Composites,” Nano Lett., 8(9), pp. 2762-2766.
[5] Zhang, M., Atkinson, K. R., and Baughman, R. H., 2004, "Multifunctional Carbon Nanotube Yarns by Downsizing an Ancient Technology," Science, 306(5700), pp. 1358-1361.

[6] Thevamaran, R., and Daraio, C., 2014, "An Experimental Technique for the Dynamic Characterization of Soft Complex Materials," Exp. Mech., 54(8), pp. 1319-1328.

[7] Thevamaran, R., Meshot, E. R., and Daraio, C., 2014, "Shock Formation and Rate Effects in Impacted Carbon Nanotube Foams," Carbon (submitted).

[8] Lattanzi, L., Raney, J. R., De Nardo, L., Misra, A., and Daraio, C., 2012 "Nonlinear Viscoelasticity of Freestanding and Polymer-Anchored Vertically Aligned Carbon Nanotube Foams,” J. Appl. Phys., 111(7), p. 074314.

[9] Zhang, Q., Lu, Y. C., Du, F., Dai, L., Baur, J., and Foster, D. C., 2010 "Viscoelastic Creep of Vertically Aligned Carbon Nanotubes," J. Phys. D.: Appl. Phys., 43(31), p. 315401.

[10] Raney, J., Fraternali, F., and Daraio, C., 2013, "Rate-Independent Dissipation and Loading Direction Effects in Compressed Carbon Nanotube Arrays," Nanotechnology, 24(25), p. 255707.

[11] Pathak, S., Lim, E. J., Abadi, P. P. S. S., Graham, S., Cola, B. A., and Greer, J. R., 2012, "Higher Recovery and Better Energy Dissipation at Faster Strain Rates in Carbon Nanotube Bundles: An In-Situ Study," ACS Nano, 6(3), pp. 2189-2197.

[12] Qiu, a., Fowler, S. P., Jiao, J., Kiener, D., and Bahr, D. F., 2011, "TimeDependent Contact Behavior Between Diamond and a CNT Turf," Nanotechnology, 22(29), p. 295702.

[13] Xu, M., Futaba, D. N., Yamada, T., Yumura, M., and Hata, K., 2010, "Carbon Nanotubes With Temperature-Invariant Viscoelasticity From $-196^{\circ}$ to $1000^{\circ} \mathrm{C}, "$ Science, $330(6009)$, pp. 1364-1368.

[14] Teo, E. H. T., Yung, W. K. P., Chua, D. H. C., and Tay, B. K., 2007, “A Carbon Nanomattress: A New Nanosystem With Intrinsic, Tunable, Damping Properties," Adv. Mater., 19(19), pp. 2941-2945.

[15] Wang, L., Ortiz, C., and Boyce, M. C., 2011, "Mechanics of Indentation Into Micro- and Nanoscale Forests of Tubes, Rods, or Pillars," ASME J. Eng. Mater. Technol., 133(1), p. 011014

[16] Hutchens, S. B., Needleman, A., and Greer, J. R., 2011, "Analysis of Uniaxial Compression of Vertically Aligned Carbon Nanotubes," J. Mech. Phys. Solids, 59(10), pp. 2227-2237.

[17] Maschmann, M. R., Ehlert, G. J., Tawfick, S., Hart, A. J., and Baur, J. W., 2014, "Continuum Analysis of Carbon Nanotube Array Buckling Enabled by Anisotropic Elastic Measurements and Modeling," Carbon N. Y., 66, pp. 377-386.

[18] Yang, X., He, P., and Gao, H., 2011, "Modeling Frequency- and TemperatureInvariant Dissipative Behaviors of Randomly Entangled Carbon Nanotube Networks Under Cyclic Loading," Nano Res., 4(12), pp. 1191-1198.

[19] Fraternali, F., Blesgen, T., Amendola, A., and Daraio, C., 2011, "Multiscale Mass-Spring Models of Carbon Nanotube Foams," J. Mech. Phys. Solids, 59(1), pp. 89-102.

[20] Raney, J. R., Fraternali, F., Amendola, A., and Daraio, C., 2011, "Modeling and In Situ Identification of Material Parameters for Layered Structures Based on Carbon Nanotube Arrays," Compos. Struct., 93(11), pp. 3013-3018.

[21] Blesgen, T., Fraternali, F., Raney, J., and Daraio, C., 2013, "Multiscale MassSpring Models of Carbon Nanotube Arrays Accounting for Mullins-Like Behavior and Permanent Deformation," Multiscale Model. Simul., 11(2), pp. $545-565$.

[22] Carretero-González, R., Khatri, D., Porter, M., Kevrekidis, P., and Daraio, C., 2009, "Dissipative Solitary Waves in Granular Crystals," Phys. Rev. Lett., $\mathbf{1 0 2}(2)$, p. 024102.

[23] Bedewy, M., Meshot, E. R., Guo, H., Verploegen, E. A., Lu, W., and Hart, A. J., 2009, "Collective Mechanism for the Evolution and Self-Termination of Vertically Aligned Carbon Nanotube Growth,” J. Phys. Chem. C, 113(48), pp. 20576-20582.

[24] Puglisi, G., and Truskinovsky, L., 2005, "Thermodynamics of RateIndependent Plasticity,” J. Mech. Phys. Solids, 53(3), pp. 655-679.

[25] Fraternali, F., Raney, J., and Daraio, C., 2013, "Modeling Microscale Instabilities in Compressed Carbon Nanotube Bundles Using Multistable Spring Models," Compos. Struct., 96, pp. 745-750.

[26] Knap, J., and Ortiz, M., 2001, "An Analysis of the Quasicontinuum Method,” J. Mech. Phys. Solids, 49(9), pp. 1899-1923.

[27] Fraternali, F., Lorenz, C. D., and Marcelli, G., 2012, "On the Estimation of the Curvatures and Bending Rigidity of Membrane Networks Via a Local Maximum-Entropy Approach," J. Comput. Phys., 231(2), pp. 528-540. 